ORIGINAL ARTICLE

\title{
POMT2 mutations cause $\alpha$-dystroglycan hypoglycosylation and Walker-Warburg syndrome
}

\author{
J van Reeuwijk, M Janssen, C van den Elzen, D Beltran-Valero de Bernabé, P Sabatelli, \\ L Merlini, M Boon, H Scheffer, M Brockington, F Muntoni, M A Huynen, A Verrips, \\ C A Walsh, P G Barth, H G Brunner, H van Bokhoven
}

See end of article for authors' affiliations

.....................

Correspondence to: Dr H van Bokhoven, Department of Human Genetics 417, Radboud University Nijmegen Medical Centre, Box 9101, 6500 HB Nijmegen, Netherlands; $\mathrm{H}$. vanBokhoven@antrg. umen.nl

Received 12 February 2005 Revised version received 18 April 2005

Accepted for publication 20 April 2005

Published Online First 13 May 2005
Background: Walker-Warburg syndrome (WWS) is an autosomal recessive condition characterised by congenital muscular dystrophy, structural brain defects, and eye malformations. Typical brain abnormalities are hydrocephalus, lissencephaly, agenesis of the corpus callosum, fusion of the hemispheres, cerebellar hypoplasia, and neuronal overmigration, which causes a cobblestone cortex. Ocular abnormalities include cataract, microphthalmia, buphthalmos, and Peters anomaly. WWS patients show defective O-glycosylation of $\alpha$-dystroglycan $(\alpha-D G)$, which plays a key role in bridging the cytoskeleton of muscle and CNS cells with extracellular matrix proteins, important for muscle integrity and neuronal migration. In $20 \%$ of the WWS patients, hypoglycosylation results from mutations in either the protein O-mannosyltransferase 1 (POMT1), fukutin, or fukutin related protein (FKRP) genes. The other genes for this highly heterogeneous disorder remain to be identified.

Objective: To look for mutations in POMT2 as a cause of WWS, as both POMT1 and POMT2 are required to achieve protein O-mannosyltransferase activity.

Methods: A candidate gene approach combined with homozygosity mapping.

Results: Homozygosity was found for the POMT2 locus at $14 \mathrm{q} 24.3$ in four of 11 consanguineous WWS families. Homozygous POMT2 mutations were present in two of these families as well as in one patient from another cohort of six WWS families. Immunohistochemistry in muscle showed severely reduced levels of glycosylated $\alpha-D G$, which is consistent with the postulated role for POMT2 in the O-mannosylation pathway.

Conclusions: A fourth causative gene for WWS was uncovered. These genes account for approximately one third of the WWS cases. Several more genes are anticipated, which are likely to play a role in glycosylation of $\alpha-D G$.
W alker-Warburg syndrome (WWS) is a congenital disorder characterised by multiple anomalies of the brain, muscle, and eye. This combination of malformations is also found in muscle-eye-brain disease (MEB) and in Fukuyama congenital muscular dystrophy (FCMD). The most severe anomalies are seen in WWS patients, especially with regard to the brain malformations. Brain malformations typical of WWS are agyria, agenesis of the corpus callosum, cerebellar vermis and septum, and occasional occipital encephalocele. ${ }^{1-3}$ The pathogenesis underlying WWS, MEB, FCMD, and other congenital muscular dystrophies (CMD) such as congenital muscular dystrophy IC (MDCIC) and ID (MDCID) involves functional disruption of $\alpha$-dystroglycan ( $\alpha$-DG) by mutations in genes that are involved in O-mannosylation of this protein. ${ }^{4-10}$ The O-linked carbohydrate chains of $\alpha$-DG are an important component of the dystrophin glycoprotein complex which mediates the interaction between the extracellular matrix and the cytoskeleton of muscle cells and neurones. ${ }^{11-15} \mathrm{~A}$ common O-mannosyl glycan structure found on $\alpha$-DG is NeuAc $\alpha 2-3$ Gal $\beta 1$-4GlcNAc $\beta 1$-2Man-Ser/Thr. ${ }^{16}{ }^{17}$ The first step in the synthesis of this glycan structure takes place in the endoplasmic reticulum by one or more proteins from a family of well conserved protein O-mannosyltransferases (PMTs). These proteins catalyse the transfer of a mannose from dolichyl phosphate activated mannose to serine or threonine residues of secretory proteins. In yeast, a family of seven PMTs (Pmtlp to Pmt7p) are known. Double and triple mutants of the PMTs in yeast indicate that proper
O-mannosylation is required for its cell wall rigidity and cell integrity. ${ }^{18}$ In Drosophila and human there are only two orthologues: POMTl and POMT2. ${ }^{16}$ RNAi knockdown of either POMT1 or POMT2 in Drosophila causes a rotation of the abdomen by $30-60^{\circ}$, demonstrating the requirement of both POMTI and POMT2 for normal muscle development in the fly. ${ }^{19}$ No embryonic nervous system or eye abnormalities were observed for POMT1 knockouts. ${ }^{20}$ In addition, it has been shown that in humans POMTl and POMT2 are also both required for O-mannosyltransferase activity. ${ }^{19} 21$ Previously we have shown that mutations in either POMT1, FCMD, or FKRP result in hypoglycosylation of $\alpha-\mathrm{DG}$, giving rise to the autosomal recessive disorder WWS..$^{22-24}$ The phenotypic similarity seen in the fly knockdown of POMT1 and POMT2 and the simultaneous requirement for both proteins to obtain O-mannosyltransferase in fly as well as human made us reconsider POMT2 as candidate gene for WWS. In this study we show that POMT2 mutations also cause WWS.

\section{METHODS}

\section{Genetic analysis}

Using standard methods we extracted DNA from peripheral blood lymphocytes. Genome-wide homozygosity mapping was carried out at our linkage facility using the $10 \mathrm{cM}$ spaced

Abbreviations: $\alpha-D G, \alpha$-dystroglycan; $C M D$, congenital muscular dystrophy; FCMD, Fukuyama congenital muscular dystrophy; MEB, muscle-eye-brain disease; PMT, protein O-mannosyltransferase; WWS, Walker-Warburg syndrome 


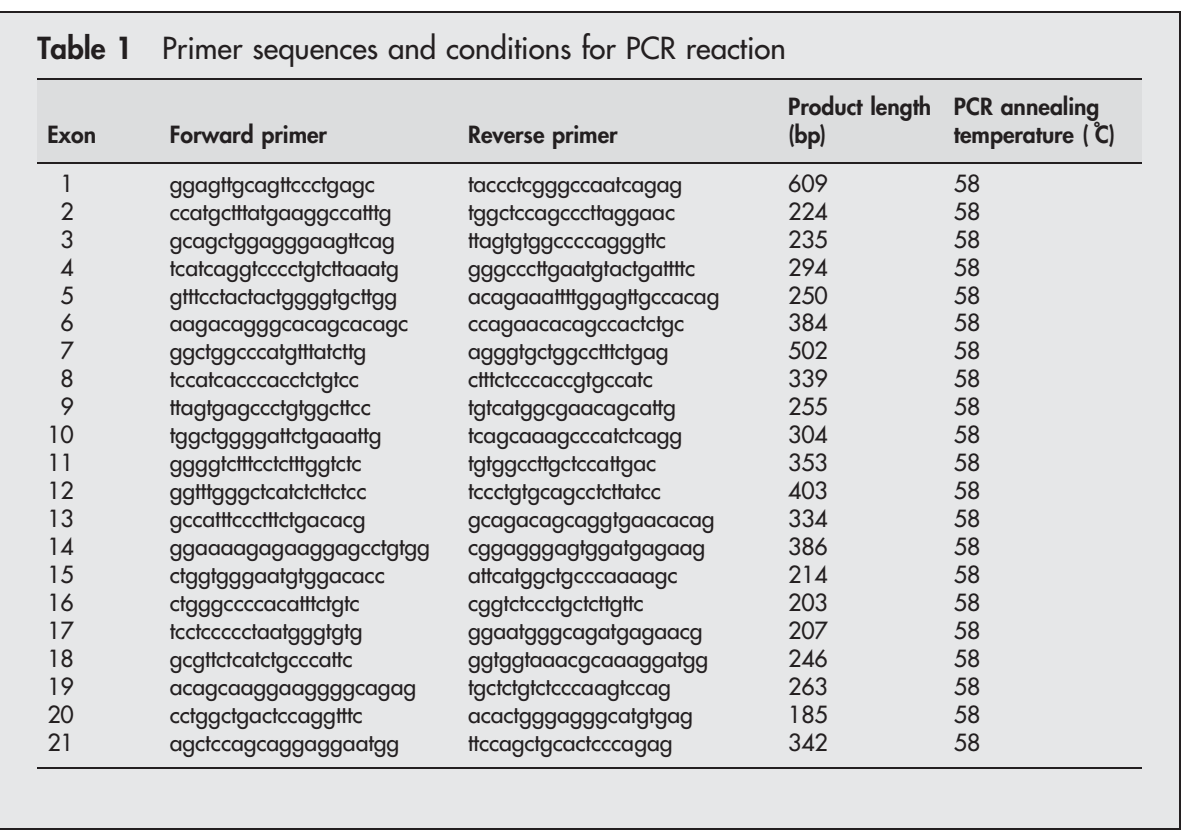

microsatellite marker set from Applied Biosystems (ABI Prism linkage mapping set version 2, Applied Biosystems, Foster City, California, USA) and at MRC geneservice in Cambridge with use of the GeneChip Mapping 10K 2.0 array from Affymetrix (Santa Clara, California, USA). Additional homozygosity mapping for POMT2 was undertaken by polymerase chain reaction (PCR) of the flanking microsatellite markers D14S279, D14S983, and D14S59, which were subsequently resolved on $8 \%$ polyacrylamide sequencing gels and developed by silver staining.

\section{Mutation analysis}

All 21 exons of the POMT2 gene were amplified using specific primers for the $5^{\prime}$ - and $3^{\prime}$-flanking intron sequences. Primers and PCR conditions are given in table 1. After purification from agarose gels, the PCR products were used for direct sequencing using the BigDye terminator kit (Perkin Elmer Applied Biosystems, Norwalk, Connecticut, USA), which were analysed on an ABI3700 capillary sequencer. The presence of the identified mutations in the normal population was tested by restriction enzyme analysis in chromosomes from control individuals. For this, the relevant amplicon was digested with TaqI $($ c.1912C $\rightarrow$ T), HpyCH4 IV $($ c.1005+lG $\rightarrow$ A), and NciI (c.126ldelC) (New England BioLabs, Beverly, Massachusetts, USA).

\section{Immunohistochemistry}

Muscle biopsies from control and WWS patients were obtained after informed consent of patients and approval of the ethics commission. Myoblast cell cultures were established by enzymatic and mechanical treatment of muscle biopsies and by plating in Dulbecco's modified Eagle's

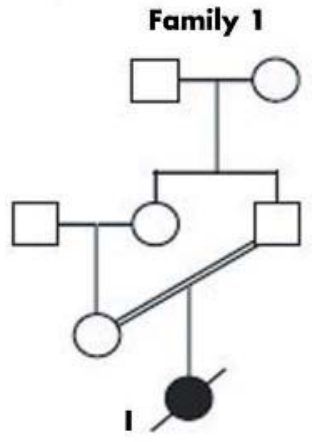

c. $1912 \mathrm{C} \rightarrow$ T, p.R638X

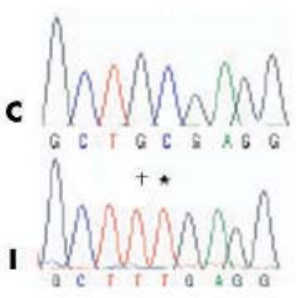

Family 2

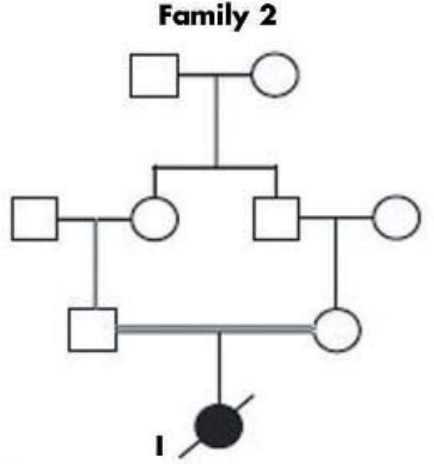

c. $1005+1 G \rightarrow A$

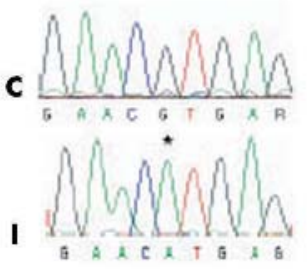

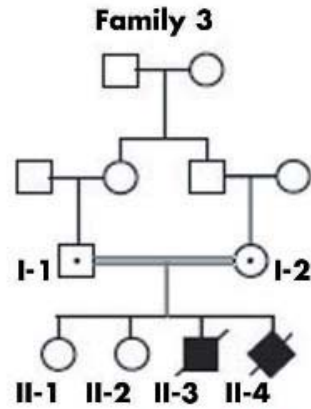

c. $1261 \mathrm{delC}$

C

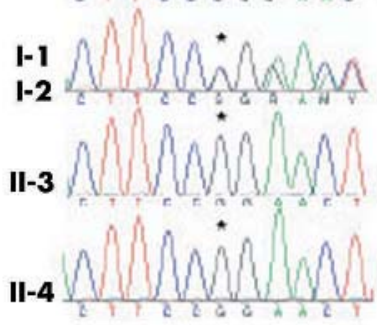

Figure 1 POMT2 mutations in families with Walker-Warburg syndrome (WWS). For each of the families with POMT2 mutations the pedigrees and DNA sequencing results are shown. Mutations are indicated with an asterisk (*). Patient 1 is homozygous for a nonsense mutation in exon 19 (c. 1912C $\rightarrow$ T, p.R638X). The mutation in this patient is preceded by a known polymorphism indicated by a dagger $(t)$ sign. Patient 2 is homozygous for a mutation in intron 8 (c. 1005+1G $\rightarrow A$ ) which is predicted ${ }^{32}$ to result in the disruption of the donor site for intron splicing of intron 8 . This mutation also disrupts a restriction site for the HpyCH4 IV enzyme and was not present in 100 chromosomes of controls. Patient 3 is homozygous for a deletion of $1 \mathrm{bp}$ in exon 12 (c. $1261 \mathrm{delC}$ ) which tends to a premature stop codon (p.T433X). The same mutation was found heterozygously in both parents and homozygously in an affected sibling in this family. 
medium plus fetal calf serum (FCS), penicillin, streptomycin, and amphotericin B (Sigma, Poole, Dorset, UK). ${ }^{25}$ Myotubes were obtained by confluent myoblast cultures allowed to differentiate for seven days in cultures medium. Samples for immunohistochemical analysis were fixed in 2\% paraformaldehyde in phosphate buffer saline (PBS) and incubated overnight with an anti- $\alpha$-dystroglycan monoclonal antibody (VIA4-1, Upstate Biotechnology, Lake Placid, New York, USA) diluted $1 / 50$, washed with PBS and then with an antimouse IgG TRITC conjugated antibody (Dako, Glostrup, Denmark). The same sample was then incubated with a polyclonal anti-caveolin 3 antibody (Santa Cruz Biotechnology, Santa Cruz, California, USA) diluted 1/50 followed by FITC conjugated anti-goat antibody. The sample was washed in PBS, mounted in Pro-long anti-fade reagent (Molecular Probes, Eugene, Oregon, USA), and examined with a Nikon epifluorescence microscope at a magnification of $\times 100$.

Immunohistochemistry on muscle biopsies was carried out as described by Jimenez-Mallebrera et al. ${ }^{26}$ In Brief, frozen $8 \mu \mathrm{m}$ sections were incubated with primary monoclonal antibodies to $\beta$-DG (NCL-b-DG, Novocastra Laboratories, Newcastle upon Tyne, UK), $\alpha$-DG (IIH6, Upstate Biotechnology), and sheep polyclonal antibody recognising the core protein of $\alpha$-DG. ${ }^{27}$ These were than revealed with an appropriate biotinylated secondary antibody (Amersham 1:200; Amersham Life Sciences, Amersham, UK) followed incubation with streptavidin conjugated to Alexa 594 (Molecular Probes) and visualised by epifluorescence microscopy.

\section{RESULTS}

Direct linkage mapping of candidate genes using genome-wide homozygosity data

We carried out genome-wide homozygosity mapping of 12 WWS patients from 11 unrelated consanguineous families in which linkage to the known WWS loci (POMT1, Fukutin, and $F K R P$ ) has been excluded. Depending on the amount of DNA available we used the $10 \mathrm{cM}$ spaced microsatellite marker set (ABI Prism linkage mapping set version 2) or the GeneChip Mapping 10K 2.0 Array (Affymetrix). As can be expected from the genetic heterogeneity of WWS, these homozygosity mapping results of mostly singletons failed to point to a single WWS locus and indeed indicated further genetic heterogeneity. Although we failed to detect POMT2 mutations in an earlier study of 24 WWS families, ${ }^{22}$ we targeted POMT2 as a candidate gene based on the premise that all three previously identified WWS genes-POMT1, fukutin and FKRP-are involved in O-mannosyl glycan synthesis. A similar function is likely for POMT2, based on the overlapping expression profiles and homologous amino acid composition compared with POMTl. In addition, recent reports indicate that POMT2 is required for the enzymatic activity of POMT1 in human as well as Drosophila. ${ }^{121}$ Our new mapping data indicated possible linkage to the POMT2 locus in four of 11 unrelated WWS families.

\section{Mutation analysis of POMT2}

Mutation analysis in all four families led to the identification of mutations in two of these, a nonsense mutation (c.1912C $\rightarrow$ T, p.R638X) in patient 1 and a splice site mutation
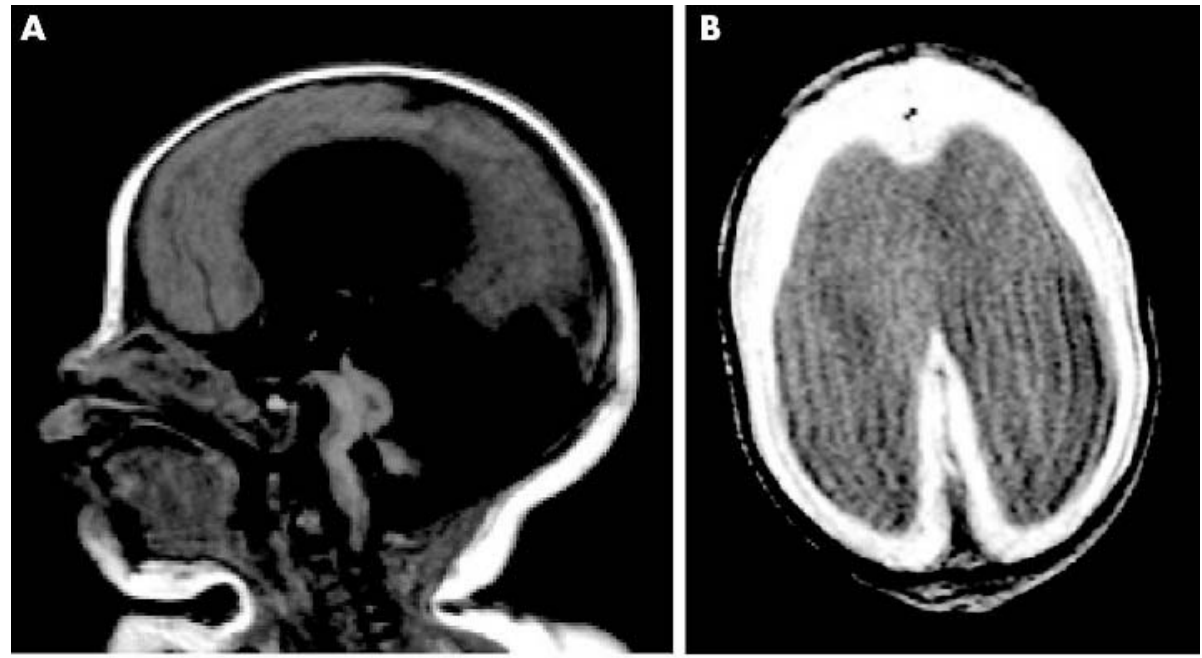

Figure 2 Brain magnetic resonance imaging of patient 1 at two days of age ( $A$ and $B$ ) and patient 2 at 16 months of age (C and D). Both patients show hydrocephalus, dilatation of the ventricles, agyria, cerebellar hypoplasia, and absence of the corpus callosum.
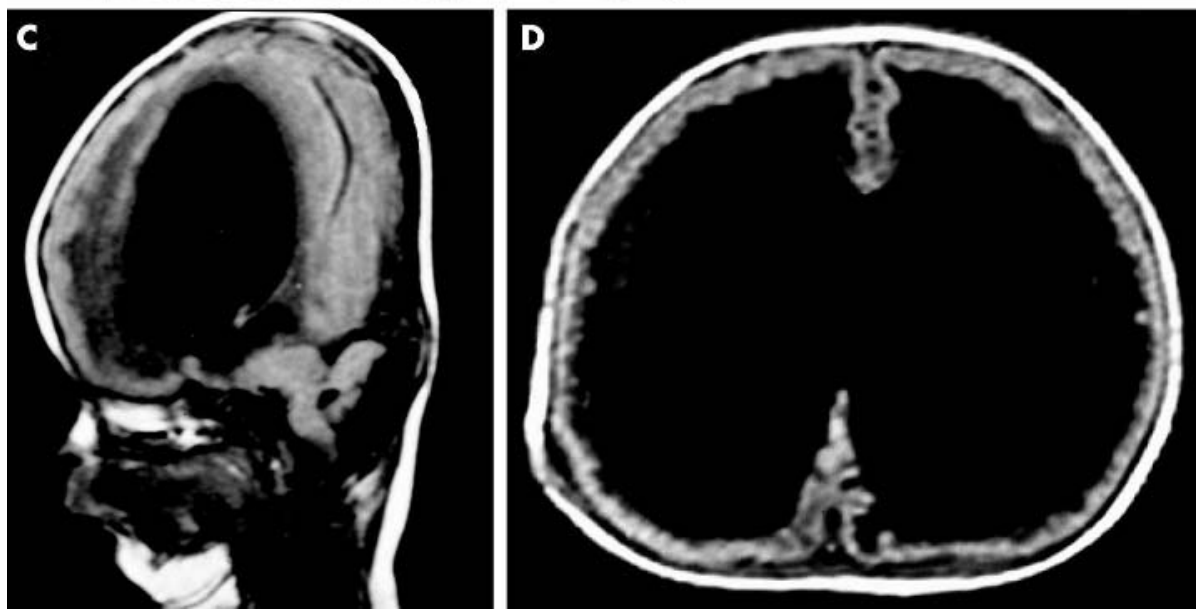

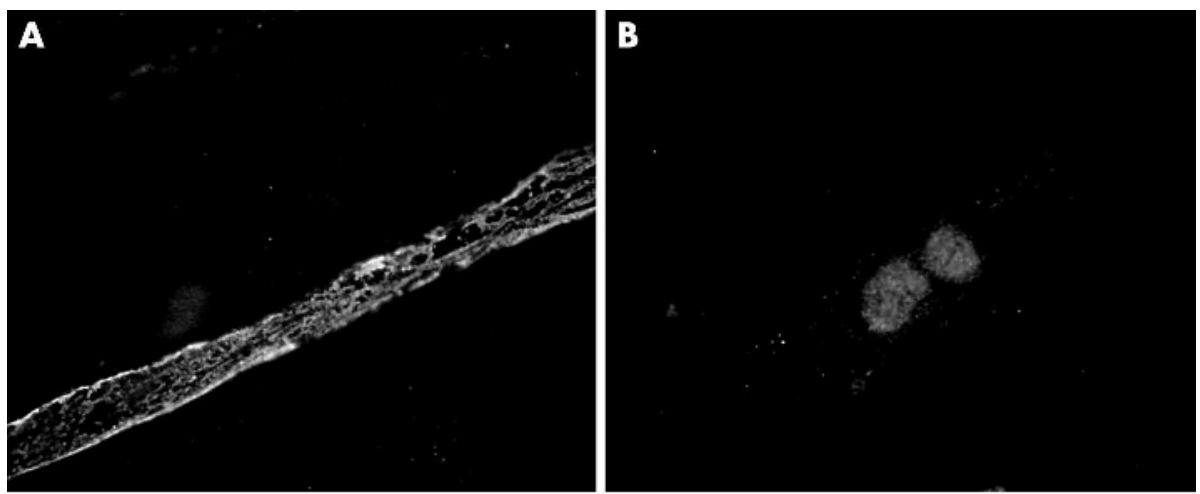

Figure 3 Immunofluorescence analysis of cultured myotubes obtained from a control individual $(A$ and $C$ ) and patient 2 ( $B$ and $D$ ). A double labelling was carried out with anti- $\alpha-D G$ glycoepitope antibody (VIA4-1) (A and B), and anti-caveolin3, a marker of muscle differentiation ( $C$ and $D)$. These were visualised using an anti-mouse TRITC conjugated secondary antibody and an anti-goat FITC conjugated antibody, respectively. In control myotube, $\alpha$-DG labelling co-localises with caveolin3 at the sarcolemma. In the WWS patient myotube, caveolin 3 was expressed with a pattern similar to the control myotube while $\alpha$-DG staining was
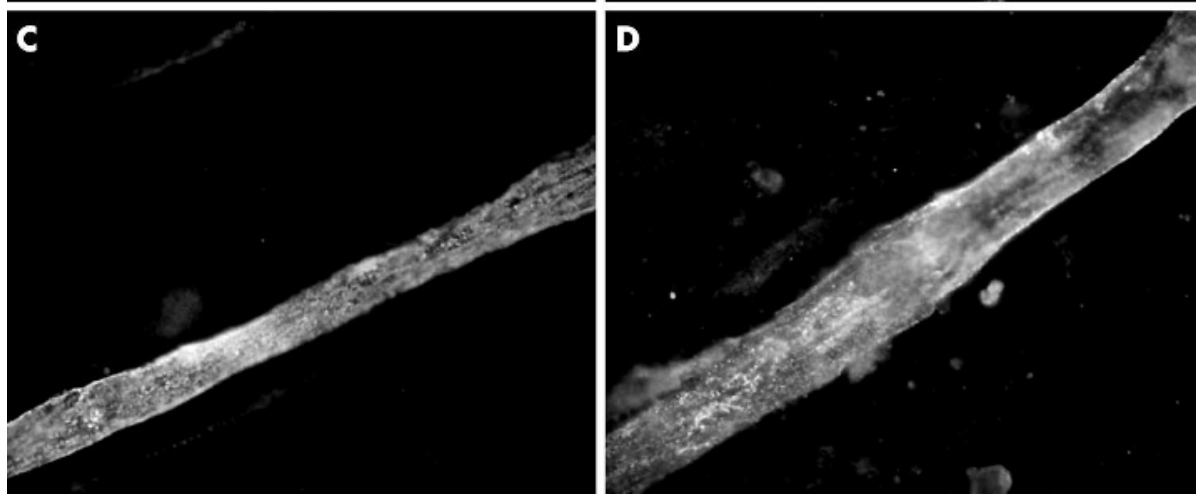
severely reduced. Magnification $\times 500$. $\alpha$-DG, $\alpha$-dystroglycan; WWS, WalkerWarburg syndrome.

(c.1005+lG $\rightarrow$ A) in patient 2, both found in homozygosity. Both mutations result in the disruption of a restriction site for Taql and HpyCH4 IV, in exon 19 and exon 8, respectively. These disruptions were not observed in control individuals digested with Taql (170 chromosomes) and HpyCH4 IV (290 chromosomes). No mutations were detected in the POMT2 exons in the other two families that showed linkage to the POMT2 locus. Reasons for this could be that their linkage was a fortuitous finding or that a mutation resides in parts of the POMT2 gene such as an intron or regulatory elements that have not been analysed. An additional six consanguineous WWS families were then tested for homozygosity at the POMT2 locus. In two of these WWS families the data were consistent with linkage to POMT2. We identified a homozygous 1 base pair (bp) deletion (c.126ldelC) in one these families. This mutation introduces a premature stop codon (p.T433X). The mutation was also homozygously present in an affected sibling and disrupts one of the two restriction sites for NciI in exon 12, which was used the verify the absence of this mutation in 140 control chromosomes from the normal population. Pedigrees and DNA sequencing results of the three POMT2 mutated families are shown in fig 1 .

\section{Clinical description WWS patients with POMT2 mutations}

Patient $\mathrm{l}$ is a girl born to uncle-niece consanguineous Moroccan parents. A prenatal ultrasound indicated hydrocephalus which was confirmed by magnetic resonance imaging, which also showed type II lissencephaly (fig 2, panels A and B). At birth at 37 weeks gestation, weight was 3150 grams, and head circumference $36 \mathrm{~cm}$. The child was severely hypotonic. Ophthalmological examination showed bilateral Peters anomaly with cataracts, left sided microphthalmia, and right sided buphthalmos. The creatine kinase was greatly raised at $12144 \mathrm{U} / \mathrm{l}$. MRI of the brain documented hydrocephalus, cobblestone lissencephaly, and aplasia of the corpus callosum.
Patient 2 is a male child born to first cousin Pakistani parents. Severe hydrocephalus was diagnosed prenatally on ultrasound and the child was delivered by caesarean section. A left sided cleft lip and palate was diagnosed. Imaging of the brain by MRI revealed aplasia of the posterior vermis, hypoplasia of the pons and cerebellum, and severe hydrocephalus and cobblestone cortex (fig 2, panels C and D). Ophthalmological investigation documented bilateral cataracts and persistent pupillary membrane. The retina could not be visualised. A muscle biopsy was consistent with a diagnosis of congenital muscular dystrophy. There was increased variability of fibre diameter, increased endomysial fibrosis, and basophilic regeneration. External genitalia were normal. The child died at the age of six months.

Patient 3 is a male infant referred at the age of two months and one week because of severe neonatal hypotonia, developmental delay, and poor visual behaviour, as previously reported.$^{26} \mathrm{He}$ was the third child of a consanguineous Bengali family. His older siblings were healthy. Congenital hydrocephalus required a shunt at two weeks of age. Serum creatine kinase was markedly raised. Ophthalmological examination showed bilateral lamellar cataracts, and buphthalmos caused by anterior chamber anomalies. He had no head control and no apparent response to visuoacoustical stimuli. Brain MRI at the age of five weeks showed severe hydrocephalus with a thin and smooth cortical mantle. The patient died at eight months of age during a respiratory infection.

A further sibling was diagnosed prenatally with severe hydrocephalus at 18 weeks' gestation. This pregnancy was subsequently terminated.

\section{Histopathology of WWS muscle tissue}

Immunolabelling of the glycoepitope of $\alpha$-DG with the VIA41 antibody $^{28}$ in cultured myotubes from patient 2 showed severely reduced staining compared with control myotubes (fig 3, panels A and B). Control labelling with anti-caveolin3, a marker of muscle differentiation, showed that $\alpha$-DG 

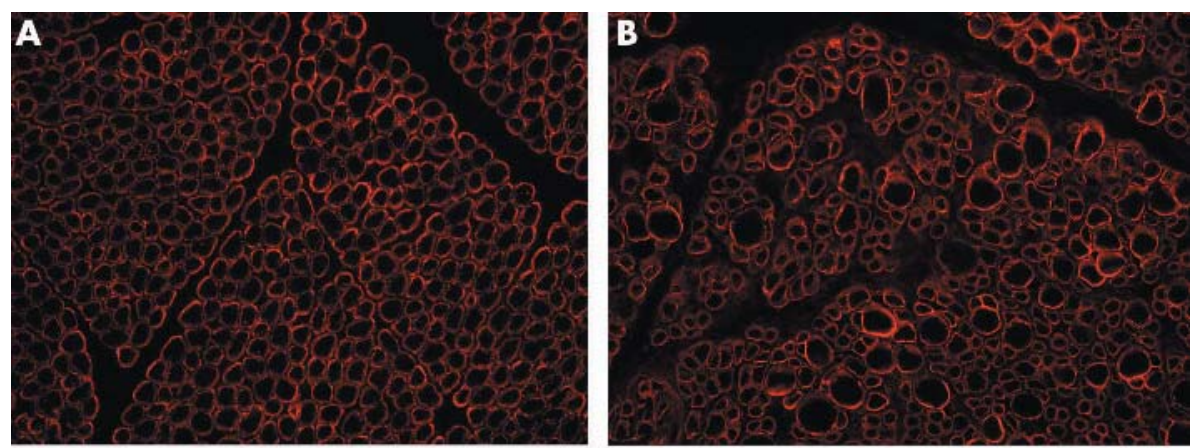

Figure 4 Muscle biopsy of control muscle $(A, C$, and $E)$ and patient $3(B$, $D$, and F). Immunostaining with antibodies to $\beta$-dystroglycan ( $\beta$-DG) was normal in both patient and control muscle (A and B). Immunostaining with antibodies that recognise core $\alpha^{-}$ dystroglycan $(\alpha-D G)(C$ and $D)$ and $a$ glycosylated epitope (IIH6) of dystroglycan (E and F) showed normal expression in control but marked reduction of staining in the patient.
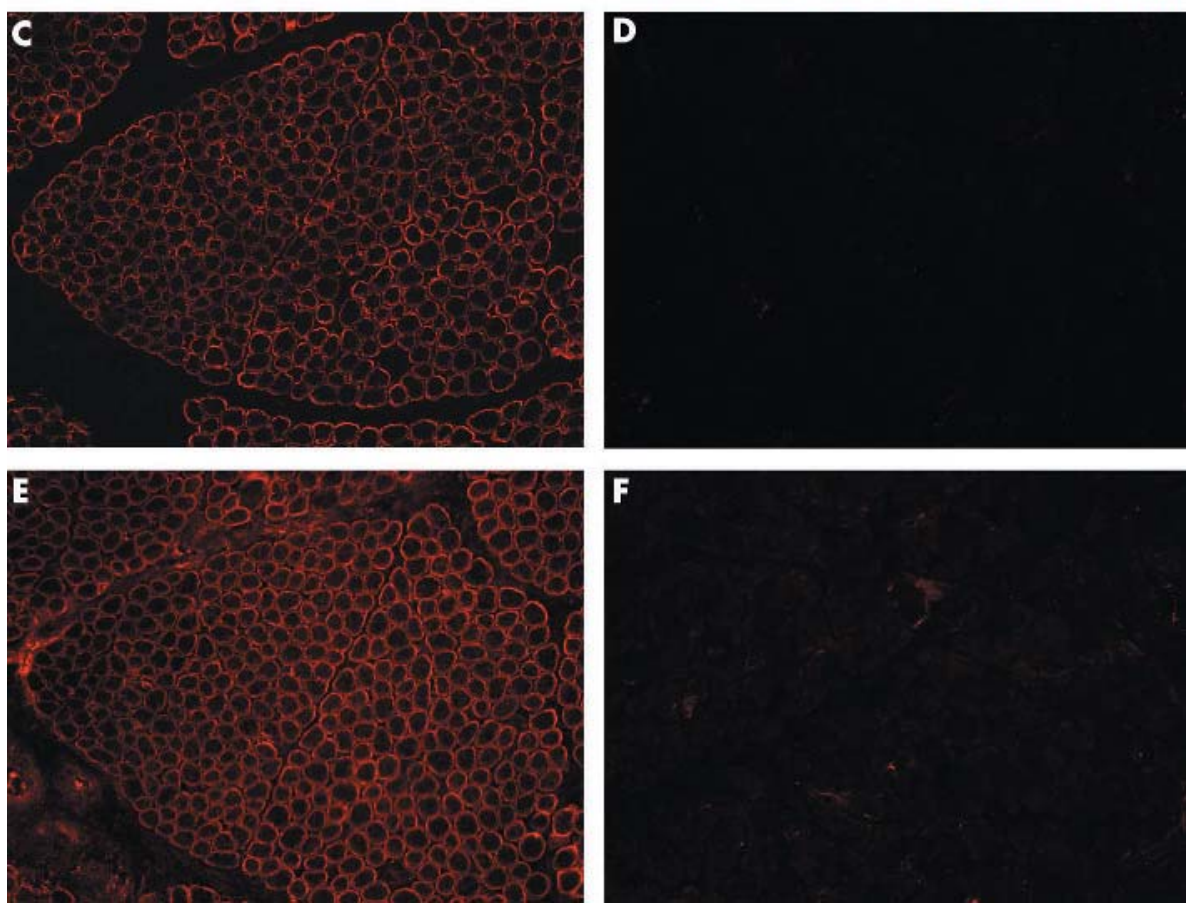

co-localises with caveolin3 at the sarcolemma of myotubes derived from a control individual (fig 3C). Similar normal staining was observed in myotubes derived from patient 2 (fig 3D). Reduced staining of $\alpha$-DG was also observed in patient 3 by immunolabelling of the core and glyco-epitope of $\alpha$-DG (fig 4, panels D and F). Normal staining was observed for $\alpha$-DG in control muscle (fig 4, panels $C$ and E). $\beta$-DG staining was normal in the patient as well as in the control (fig 4, panels A and B). Reduction of immunolabelling of $\alpha$ DG and a mild reduction of laminin- $\alpha 2$ in muscle tissue from patient 3 were described previously by Jiminez-Mallebrera et al. ${ }^{26}$ No muscle tissue was available for patient 1 .

\section{DISCUSSION}

We have detected three homozygous mutations in POMT2 in three families with typical WWS. The mutations included a nonsense mutation resulting in a premature stop codon, a splice site mutation, and a $\mathrm{l}$ bp deletion leading to a premature stop codon. All mutations were homozygous in the patients (fig 1). The phenotype seen in these WWS patients is indistinguishable from that of patients with POMT1, FCMD, or FKRP mutations. One of the sibpair from family 3 who carries a homozygous 1 bp deletion mutation was described earlier, and hypoglycosylation of $\alpha$-DG (fig 4 ) and a possible reduction of laminin- $\alpha 2$ in muscle tissue was documented. ${ }^{26}$ Absence of glycosylated $\alpha$-DG was also seen in a muscle biopsy from patient 2 in the present study (fig 3).
This is consistent with the postulated role of O-glycosylation in normal neuromuscular, brain, and eye development. ${ }^{11} 29$

Although previously no enzymatic activity of POMT1 and POMT2 was determined in vertebrates, the involvement of POMTl in WWS, the high amino acid identity between the two paralogues, and the overlapping expression pattern was reason to hypothesise that mutations in POMT2 also give rise to WWS. ${ }^{30}$ For this reason we previously undertook mutation analysis of POMT2 in 24 unrelated patients but no causative mutations were detected. ${ }^{22}$ In this study, 17 additional families where investigated, of which six showed possible linkage to the POMT2 locus. We found POMT2 mutations in three of these (fig 1), resulting in a frequency of 7\% (3 of 41 families). Thus the incidence of POMT2 mutations appears to be in the same range as that of POMTI. POMTI mutations were previously detected at a frequency of $20 \%$ and $7 \%$ in two large samples of WWS patients. ${ }^{22}{ }^{31}$ This is in accordance with the requirement of both proteins to obtain O-mannosyltransfererase activity. ${ }^{19} 21$ So far, mutations in POMT1, POMT2, fukutin, and FKRP together explain almost one third of the WWS patients in our cohort. The majority of WWS cases remain unexplained and further genetic heterogeneity is likely from our genome-wide homozygosity data.

\section{ACKNOWLEDGEMENTS}

We thank the families for contributing material for this study, MRC Geneservice Cambridge and our linkage facility for the genome-wide homozygosity mapping, and C Jimenez-Mallebrera for help with 
immunohistochemical analysis. This work was supported by grants from the "Prinses Beatrix Fonds" (MAR02-117), the Dutch foundation for Scientific Research (NWO, 903-42-190), "Hersenstichting Nederland" (11F503.21), the Netherlands Genomics Initiative (Horizon programme), Fondazione Carisbo, Italy, Stichting Spieren voor Spieren, and also the Muscular Dystrophy Campaign grant to FM is gratefully acknowledged.

\section{Authors' affiliations}

J van Reeuwijk, M Janssen, C van den Elzen, D B-V de Bernabé*, H Scheffer, H G Brunner, H van Bokhoven, Department of Human Genetics, Radboud University Nijmegen Medical Centre, Nijmegen, Netherlands

M A Huynen, CMBI (Centre for Molecular and Biomolecular Informatics), NCLMS (Nijmegen Centre for Molecular Life Sciences), Radboud University Niimegen Medical Centre

A Verrips, Department of Neurology, Canisius Wilhelmina Hospital, Nijmegen

P Sabatelli, ITOI (Istituto per i Trapianti d'Organo e l'Immunocitologia), Consiglio Nazionale delle Ricerche, c/o Istituto Ortopedico Rizzoli, Bologna, Italy

L Merlini, Neuromuscular Unit, Istituto Ortopedico Rizzoli

M Boon, Department of Neurology, University Medical Centre Groningen, Groningen, Netherlands

M Brockington, F Muntoni, Dubowitz Neuromuscular Centre, Imperial College, Hammersmith Campus, London, UK

C A Walsh, Howard Hughes Medical Institute and Department of Neurology, Beth Israel Deaconess Medical Center, Boston,

Massachusetts, USA

P G Barth, Department of Paediatric Neurology, Academic Medical Centre, Emma Childrens' Hospital AMC, University of Amsterdam, Amsterdam, Netherlands

*Present address: Howard Hughes Medical Institute, Department of Physiology and Biophysics, University of lowa, lowa City, lowa, USA Competing interests: none declared

\section{REFERENCES}

1 Dobyns WB, Pagon RA, Armstrong D, Curry CJ, Greenberg F, Grix A, Holmes LB, Laxova R, Michels VV, Robinow M. Diagnostic criteria for WalkerWarburg syndrome. Am J Med Genet 1989:32:195-210.

2 Cormand B, Pihko H, Bayes M, Valanne L, Santavuori P, Talim B, GershoniBaruch R, Ahmad A, van Bokhoven H, Brunner HG, Voit T, Topaloglu H, Dobyns WB, Lehesjoki AE. Clinical and genetic distinction between WalkerWarburg syndrome and muscle-eye-brain disease. Neurology 2001:56:1059-69.

3 van Reeuwijk J, Brunner HG, van Bokhoven H. Glyc-O-genetics of WalkerWarburg syndrome. Clin Genet 2005;67:281-9.

4 Martin-Rendon E, Blake DJ. Protein glycosylation in disease: new insights into the congenital muscular dystrophies. Trends Pharmacol Sci 2003;24: 178-83.

5 Martin PT, Freeze HH. Glycobiology of neuromuscular disorders. Glycobiology 2003;13:67-75R.

6 Grewal PK, Hewitt JE. Glycosylation defects: a new mechanism for muscular dystrophy? Hum Mol Genet 2003;12(Spec No 2):R259-64

7 Muntoni F, Brockington M, Torelli S, Brown SC. Defective glycosylation in congenital muscular dystrophies. Curr Opin Neurol 2004;17:205-9.

8 Endo T, Toda T. Glycosylation in congenital muscular dystrophies. Biol Pharm Bull 2003;26:1641-7.

9 Dalkilic I, Kunkel LM. Muscular dystrophies: genes to pathogenesis. Curr Opin Genet Dev 2003;13:231-8.

10 Schachter H, Vajsar J, Zhang W. The role of defective glycosylation in congenital muscular dystrophy. Glycoconj J 2004;20:291-300.

11 Michele DE, Barresi R, Kanagawa M, Saito F, Cohn RD, Satz JS, Dollar J, Nishino I, Kelley RI, Somer H, Straub V, Mathews KD, Moore SA, Campbell KP. Post-translational disruption of dystroglycan-ligand interactions in congenital muscular dystrophies. Nature 2002;418:417-22.
12 Martin PT. Dystroglycan glycosylation and its role in matrix binding in skeletal muscle. Glycobiology 2003;13:55-66.

13 Kim DS, Hayashi YK, Matsumoto H, Ogawa M, Noguchi S, Murakami N, Sakuta R, Mochizuki M, Michele DE, Campbell KP, Nonaka I, Nishino I. POMTl mutation results in defective glycosylation and loss of laminin-binding activity in alpha-DG. Neurology 2004;62:1009-11.

14 Kanagawa M, Saito F, Kunz S, Yoshida-Moriguchi T, Barresi R, Kobayashi YM, Muschler J, Dumanski JP, Michele DE, Oldstone MB, Campbell KP. Molecular recognition by LARGE is essential for expression of functional dystroglycan. Cell 2004;117:953-64.

15 Michele DE, Campbell KP. Dystrophin-glycoprotein complex: posttranslational processing and dystroglycan function. J Biol Chem 2003;278:15457-60

16 Willer T, Valero MC, Tanner W, Cruces J, Strahl S. O-mannosyl glycans: from yeast to novel associations with human disease. Curr Opin Struct Biol 2003;13:621-30.

17 Endo T. O-mannosyl glycans in mammals. Biochim Biophys Acta 1999; 1473:237-46.

18 Gentzsch $M$, Tanner W. The PMT gene family: protein O-glycosylation in Saccharomyces cerevisiae is vital. EMBO J 1996;15:5752-9.

19 Ichimiya T, Manya H, Ohmae Y, Yoshida H, Takahashi K, Ueda R, Endo T, Nishihara S. The twisted-abdomen phenotype of drosophila POMT1 and POMT2 mutants coincides with their heterophilic protein Omannosyltransferase activity. J Biol Chem 2004;279:42638-47.

20 Martin-Blanco E, Garcia-Bellido A. Mutations in the rotated abdomen locus affect muscle development and reveal an intrinsic asymmetry in Drosophila. Proc Natl Acad Sci USA 1996;93:6048-52.

21 Manya H, Chiba A, Yoshida A, Wang X, Chiba Y, Jigami Y, Margolis RU, Endo T. Demonstration of mammalian protein O-mannosyltransferase activity: coexpression of POMT1 and POMT2 required for enzymatic activity. Proc Natl Acad Sci USA 2004;101:500-5.

22 Beltran-Valero de Bernabe D, Currier S, Steinbrecher A, Celli J, van Beusekom E, van der Zwaag B, Kayserili H, Merlini L, Chitayat D, Dobyns WB, Cormand B, Lehesjoki AE, Cruces J, Voit T, Walsh CA, van Bokhoven H, Brunner HG. Mutations in the O-mannosyltransferase gene POMT1 give rise to the severe neuronal migration disorder Walker-Warburg syndrome. Am J Hum Genet 2002:71:1033-43.

23 de Bernabe DB, van Bokhoven H, van Beusekom E, Van den Akker W, Kant S, Dobyns WB, Cormand B, Currier S, Hamel B, Talim B, Topaloglu H, Brunner HG. A homozygous nonsense mutation in the fukutin gene causes a Walker-Warburg syndrome phenotype. J Med Genet 2003;40:845-8.

24 Beltran-Valero de Bernabe D, Voit T, Longman C, Steinbrecher A, Straub V, Yuva Y, Herrmann R, Sperner J, Korenke C, Diesen C, Dobyns WB, Brunner HG, van Bokhoven H, Brockington M, Muntoni F. Mutations in the FKRP gene can cause muscle-eye-brain disease and Walker-Warburg syndrome. J Med Genet 2004;41:e61.

25 Cenni V, Sabatelli P, Mattioli E, Marmiroli S, Capanni C, Ognibene A, Squarzoni S, Maraldi NM, Bonne G, Columbaro M, Merlini L, Lattanzi G. Lamin A N-terminal phosphorylation is associated with myoblast activation: impairment in Emery-Dreifuss muscular dystrophy. J Med Genet 2005;42:214-20

26 Jimenez-Mallebrera C, Torelli S, Brown SC, Feng L, Brockington M, Sewry CA, Beltran-Valero dB, Muntoni F. Profound skeletal muscle depletion of alphadystroglycan in Walker-Warburg syndrome. Eur I Paediatr Neurol 2003;7:129-37.

27 Herrmann R, Straub V, Blank M, Kutzick C, Franke N, Jacob EN, Lenard HG, Kroger S, Voit T. Dissociation of the dystroglycan complex in caveolin-3deficient limb girdle muscular dystrophy. Hum Mol Genet 2000;9:2335-40.

28 Ervasti JM, Campbell KP. Membrane organization of the dystrophinglycoprotein complex. Cell 1991;66:1121-31.

29 Moore SA, Saito F, Chen J, Michele DE, Henry MD, Messing A, Cohn RD, Ross-Barta SE, Westra S, Williamson RA, Hoshi T, Campbell KP. Deletion of brain dystroglycan recapitulates aspects of congenital muscular dystrophy. Nature 2002;418:422-5.

30 Willer T, Amselgruber W, Deutzmann R, Strahl S. Characterization of POMT2, a novel member of the PMT protein O-mannosyltransferase family specifically localized to the acrosome of mammalian spermatids. Glycobiology 2002;12:771-83.

31 Currier SC, Lee CK, Chang BS, Bodell AL, Pai GS, Job L, Lagae LG, Al Gazali LI, Eyaid WM, Enns G, Dobyns WB, Walsh CA. Mutations in POMT1 are found in a minority of patients with Walker-Warburg syndrome. Am J Med Genet A 2005;133A:53-7.

32 Reese MG, Eeckman FH, Kulp D, Haussler D. Improved splice site detection in Genie. J Comput Biol 1997;4:31 1-23. 